\title{
VARIABILITY IN RIGHT HEMISPHERE LANGUAGE FUNCTION AFTER CALLOSAL SECTION: EVIDENCE FOR A CONTINUUM OF GENERATIVE CAPACITY ${ }^{1}$
}

\author{
JOHN J. SIDTIS, ${ }^{*}{ }^{2}$ BRUCE T. VOLPE,* DONALD H. WILSON, \\ GAZZANIGA*
}

*Division of Cognitive Neuroscience, Cornell University Medical College, New York, New York 10021; $\ddagger$ Department of Neurosurgery, Dartmouth-Hitchcock Medical Center, Hanover, New Hampshire 03755; and ${ }^{\S}$ Department of Neurosurgery, Medical College of Ohio, Toledo, Ohio 43699

\begin{abstract}
Two recent patients in our behavioral series investigating the psychological effects of callosal section exhibit right hemisphere language. Using lateralized visual and auditory stimulation, semantic, phonetic, and expressive linguistic functions were examined. While the right hemisphere language systems in both patients were shown to be capable of semantic information processing, they differed in their abilities to process phonetic information, follow verbal commands, and produce linguistic responses. It is argued that the differences between left and right hemisphere language systems are quantitative and are best characterized along a continuum of generative capacity. It is the variability in such capacity that appears to be responsible for the variability in right hemisphere language function within the split-brain population.
\end{abstract}

The problem of characterizing the nature and extent of right hemisphere language function has fostered a great deal of interest and an even greater amount of speculation (for reviews, see Hecaen, 1978; Hecaen and Albert, 1978). One of the sources that has provided the most dramatic observations has been the study of the linguistic capacities of the separated right hemisphere in subjects who have undergone surgical section of the corpus callosum for seizure control. Although it has been shown that only a few of these split-brain patients possess a significant right hemisphere language capacity, such subjects have provided a means by which the characteristics of right hemisphere language systems can be examined in relative isolation from that observed in the left hemisphere.

There has been a good deal of agreement with respect to several characteristics of right hemisphere language across both callosal sectioned subjects and methods of evaluation. The most striking difference between the hemispheres lies in the domain of linguistic expression. While the left hemisphere mediates expressive language, the right hemisphere is generally only capable of com-

\footnotetext{
1 This work was supported by United States Public Health Service Grant NS15053-02, the Alfred P. Sloan Foundation, and the McKnight Foundation. We wish to thank Dr. Jeffrey D. Holtzman for his detailed comments on this paper.

${ }^{2}$ To whom correspondence should be addressed at Division of Cognitive Neuroscience, Department of Neurology, Cornell University Medical College, 1300 York Avenue, New York, NY 10021.
}

prehension (Gazzaniga and Sperry, 1967), which is limited in several areas. Right hemisphere language comprehension has been shown to be strongest for nouns (Gazzaniga and Hillyard, 1971), and although it has been suggested that the comprehension of verbs is similar to that found for nouns when word frequency is taken into account (Zaidel, 1976a), the ability to carry out verbal commands is grossly deficient (Gazzaniga and Hillyard, 1971). Moreover, there is little or no capacity for syntactic processing in the right hemisphere (Gazzaniga and Hillyard, 1971; Zaidel, 1977). In general, when linguistic function has been demonstrated in the separated right half brain, performance suggests a capacity limited to lexical access.

This general view of the language capacity of the right hemisphere can be contrasted with the observations made on another callosum-sectioned patient in our series, P. S. In addition to the previously described receptive skills, this patient's right hemisphere was also found to have a significant expressive capacity. Initially, this was observed as an ability to spell using Scrabble letters and to direct the left hand in writing. The patient could also carry out verbal commands lateralized to the right hemisphere (Gazzaniga et al., 1977). Moreover, right hemisphere language capacity did not remain stable but increased during the postoperative course, apparently culminating in the ability to access the speech system (Gazzaniga et al., 1979).

One of the main issues emerging from the study of language in the callosally disconnected right hemisphere 
has been whether the differences between this linguistic system and that of the left hemisphere are of a qualitative or quantitative nature. The argument for qualitative differences has been based largely on an apparent deficit in phonetic processing observed in patients from the earlier Bogen and Vogel series (Levy and Trevarthen, 1977; Zaidel, 1976b). However, in the patient who has been shown to possess the most extensive right hemisphere language capability within the split-brain population (P. S.), qualitative differences have not been observed (Gazzaniga et al., 1979; Gazzaniga and LeDoux, 1978; Gazzaniga et al., 1977). Two recent patients in our behavioral series provide examples of the variability in the extent to which language capacity is found in the callosally disconnected right hemisphere. The present report describes several aspects of their right hemisphere language systems that suggest that generative capacity may be a critical factor in such variability. The term generative is used here to characterize an ability to use information conveyed by the stimulus. In terms of expressive language, such capacity is most obviously reflected in speaking and writing but also is seen as important in nonlinguistic responses, such as pointing, when linguistic judgments are expressed. In terms of receptive language, generative capacity cannot be observed directly, but may be inferred on the basis of performance on tasks that are believed to require synthesis in the processing of available information. The system mediating language in the right hemisphere, then, is seen as a qualitatively similar, albeit quantitatively limited, version of the left hemisphere system. Moreover, the extent of quantitative difference between the hemispheres appears to be widely variable across individuals.

\section{Visual Word Comprehension}

In previous commissurotomy patients, right hemisphere comprehension has been demonstrated as an ability to recognize picture-word and word-picture correspondence. The present evaluation was carried out to examine further semantic processing capability by determining the relative performance of each hemisphere in recognizing relationships such as synonymy, antonymy, class membership, and functional relatedness based on information conveyed through visually presented words. The ability of each hemisphere to recognize the meaning of verbs was also examined.

\section{Method̀s \\ Subjects}

$V . P . \mathrm{V} . \mathrm{P}$. is a 27 -year-old right-handed female who suffered from a seizure disorder that could not be managed with antiepileptic drugs. She underwent the twostage microneurosurgical section of her corpus callosum. In April of 1979, the anterior body, genu and rostrum of the callosum were sectioned. Seven weeks later, the callosal section was completed by dividing the remaining body and splenium.

V. P. was the product of a full term pregnancy and uncomplicated delivery. She had no unusual illnesses in childhood and reached landmarks normally. There was no family history of neurological disorders. At age six, she experienced recurrent seizures following febrile illnesses that included measles and scarlet fever. The disorder was characterized by "minor spells," and rare generalized convulsions. Anticonvulsant medicine controlled the seizure disorder and she graduated from high school. The EEG records from this period revealed spike and slow wave activity diffusely. By 1976 , she was experiencing episodes of blank staring lasting for seconds, occurring several times per day. EEG records during this period revealed bilateral 4-cps spike and slow wave activity and excessive sharp activity with left temporal predominance. In 1979, she was experiencing grand mal, petit mal, and myoclonic episodes while on multiple anticonvulsants. EEG records documented the deterioration. Further medical management could not control her mixed seizure disorder. She was referred for further management to Dr. Mark Rayport at the Medical College of Ohio. Evaluation included a normal skull film, CT scan, and angiogram. She underwent partial anterior callosal section in early April 1979, and the resection of her callosum was completed in a second operation 7 weeks later.

On neurological exam 4 months after her final operation, she was alert, oriented, and conversed easily about present and past events. She could read and write and had no difficulty with the standard clinical probes of higher integrative function. However, she displayed several of the classically described symptoms of the splitbrain syndrome (Gazzaniga, 1970). For example, she was often clumsy when performing acts requiring control of distal left limb musculature after verbal command. She had no difficulty manipulating or using objects. When blindfolded, generally she could not describe objects placed out of vision in her left hand, although she could pick them from an array. Blindfolded again, she had consistent difficulty describing proprioceptive testing on the left. When asked to mimic with her left index finger whether her toe was up or down, she was quite accurate. Cranial nerve testing revealed similar phenomena. She seemed to have a complete left homonymous hemianopia. However, when asked to mimic what she saw in her left visual field with her left hand she was very accurate. Generally, when the sensorimotor functions discretely subserved by the right hemisphere were tested in the usual manner by verbal report, she appeared to have deficits. However, by changing the approach, we found that she had a completely normal neurological exam.

$J$. W. J. W. is a 26-year-old right-handed male who suffered from intractable epilepsy since 1972. He underwent the two-stage microneurosurgical section of his corpus callosum over the summer and fall of 1979. For a detailed description of the surgical technique, see Wilson et al. (1978).

J. W. was the product of a full term pregnancy and uncomplicated delivery who reached normal psychomotor milestones. There was no family history of neurologic disorder. At the age of 13, after concussive head trauma without skull fracture, he began to experience infrequent episodes of absence spells. There were no tonic/clonic movements or other unusual behavior noted with these spells, and they were not treated. He graduated from high school at age 18. One year after graduation, he 
experienced a major motor seizure. A complete neurological evaluation, including an LP, brain scan, skull films, and blood chemistries, yielded normal results. An EEG revealed irregular polyspike and high voltage repetitive 3-cps spike and wave bursts during sleep. These abnormalities had a right anterior temporal prominence. Over the next 7 years, he had multiple hospitalizations during which attempts were made to manage his epilepsy. In spite of adequate serum levels of antiepileptic medication, he suffered frequent major motor seizures. The EEGs revealed irregular polyspike and spike and wave with occasional 3-cps activity bilateral and anterior. A CT scan was normal. During 1977 through 1979, he continued to have frequent episodes of grand mal epilepsy and many petit mal attacks each day while on adequate therapeutic doses of several different medications. He was referred to Dr. Donald H. Wilson of the Dartmouth-Hitchcock Medical Center. On neurological exam 8 months after his second operation, he was oriented, alert, and conversed easily about present and past events. Testing of higher integrative function was normal. With the exception of split-brain phenomena similar to that described for V. P., his neurological exam was normal.

\section{Materials and procedures}

Visual stimuli were generated on a $48-\mathrm{cm}$ video screen by an Apple II microprocessor which also controlled exposure duration $(150 \mathrm{msec})$ and lateral position $\left(1.6^{\circ}\right.$ to the left or right of a center fixation point). Five tests were constructed to evaluate the recognition of the following semantic relationships: synonym (e.g., boatship), antonym (e.g., day-night), function (e.g., clocktime), superordinate category membership (e.g., lakewater), and subordinate category membership (e.g., tree-oak). Each test consisted of 25 three (subtending $1.5^{\circ}$ of visual angle) to five (subtending $2.5^{\circ}$ ) uppercase letter words presented in random order to each visual field. All words were rated with a frequency of at least 50 per million (A) on the Thorndike-Lorge (1944) word count and most were rated with a frequency of at least 100 per million (AA). Additional related and distractor words were used for the construction of response cards.

Prior to each test, subjects were provided with instructions and examples of the type of judgment required. Both subjects had participated in testing previously and thus were familiar with the general procedure. A typical trial would begin with the instruction to fixate on an asterisk located at the center of the video screen. After fixation was established by the subject and maintained for a short interval, a word would be presented to either the left or right of the visual midline. Following this lateralized presentation, the subject was provided with a response card containing four words. The subject was asked to point to the word related to the previous stimulus by the specified rule (e.g., means the opposite). On each trial, the subject was asked not to name the stimulus, but rather to respond with the hand homologous to the field of stimulation (e.g., a left field word was responded to with the left hand). This response arrangement exploits the fact that each hemisphere exerts primary control over the contralateral distal extremity (Gaz- zaniga et al., 1967). Eye movements were monitored during testing with a video camera fitted with a 10:1 remote control zoom lens.

The verbs test was in the same general form, except that, following the lateralized presentation of one of six common verbs (write, play, strike, pour, drink, and eat), the patient was asked to demonstrate the meaning of the stimulus word using any of the 12 items placed on the table using the hand homologous to the field of stimulation.

\section{Results and Discussion}

Left and right hemisphere recognition scores on each test of semantic relationship are presented for both subjects in Table I. Overall, V. P. was more arcurate than J. $\mathrm{W}$. due to her higher right hemisphere performance. For both subjects, however, right hemisphere recognition was significantly greater than chance (binomial $p<0.05$ ) on all tests. A similar pattern was observed for the verbs test, on which each subject's left hemisphere score was $100 \%$. Right hemisphere performance was somewhat lower at $83.3 \%$ for V. P. and $75.0 \%$ for J. W., but, in each instance, it was significantly greater than chance (binomial $p<0.05$ ).

The results of the semantic relationship tests demonstrate the facility of the right hemisphere in semantic information processing. The relatively poorer performance of the right hemisphere may be due to any or all of several factors. The right hemisphere may have a smaller lexicon than that found in the left, it may be less proficient at visual word processing, or it may have a greater difficulty in responding to verbal material.

At least in the domain of high frequency words, the possibility that the right hemisphere's lexicon is smaller than that found in the left appears inappropriate in view of recent findings demonstrating that semantic activation in one callosally disconnected hemisphere has a significant facilitatory effect on subsequent activation of related information in the other hemisphere (J. J. Sidtis, J. D. Holliman, B. T. Volpe, D. H. Wilson, and M. S. Gazzaniga, manuscript in preparation). Thus, in the range of lexical items used in the present tests, the language systems in each hemisphere appear to access a functionally common semantic network. Hemispheric differences in other components of the comprehension

TABLE I

Left and right hemisphere accuracy scores on five tests of semantic relationship obtained from two subjects who have undergone complete section of the corpus callosum

Each percentage is based on at least 23 trials.

\begin{tabular}{lcccc}
\hline & \multicolumn{2}{c}{ V.P. } & \multicolumn{3}{c}{ J. W. } \\
\cline { 2 - 6 } Semantic Relationship & \multicolumn{4}{c}{ \% correct } \\
\cline { 2 - 6 } & Left & Right & \multicolumn{3}{c}{ Left } & Right \\
\hline \multirow{2}{*}{ Synonym } & 96.0 & 80.0 & 87.0 & 43.5 \\
Antonym & 92.3 & 70.8 & 100.0 & 62.5 \\
Function & 100.0 & 84.0 & 100.0 & 66.7 \\
Superordinate & 96.0 & 80.0 & 96.0 & 68.0 \\
Subordinate & 95.8 & 80.8 & 100.0 & 72.0 \\
& & & & \\
Mean & 96.0 & 79.2 & 96.7 & 62.8 \\
\hline
\end{tabular}


process, such as lexical access, may account for the observed performance asymmetries, as may differences in the ability to respond to linguistic information. It is likely that both alternatives contribute to the observed hemispheric differences. In the next section, it will be argued that the limiting factor in both linguistic expression and comprehension is the degree of generative capacity found in the right hemisphere.

With respect to the verbs test, the present results provide further evidence that the right hemisphere can comprehend verbs as well as nouns. However, as will be discussed in part III, the ability to carry out the praxic component of verbal commands constitutes part of a generative capacity that does not necessarily accompany receptive function.

\section{Phonetic Processing in the Right Hemisphere}

Despite the general agreement regarding the right hemisphere's ability to access meaning, there is little consensus about how such access is gained. Based on the right hemisphere's apparent inability to generate rhyme in response to target stimuli (Levy and Trevarthen, 1977), some reports have suggested that particular language functions are mediated by qualitatively different processes in each hemisphere. Specifically, it has been claimed that the right hemisphere analyzes spoken language through some unspecified "acoustic-Gestalt" process and written language through ideographic interpretation. Phonetic analysis is said not to play a role in either hypothetical process since the capacity for such analysis is supposed to be restricted to the left hemisphere (Levy and Trevarthen, 1977; Zaidel, 1976a). The present section evaluates the right hemisphere's capacity for making auditory phonetic discriminations and for recognizing phonetic relationships (rhyme) in visually presented information.

\section{Methods}

\section{Materials and procedures}

The standard dichotic consonant-vowel identification test is identical to the one used in previous studies of commissurotomized subjects (Springer and Gazzaniga, 1975; Springer et al., 1978). It consisted of pairs of natural speech syllables selected from among the following six: $/ b a, d a, g a, p a, t a, k a /$. Each member of a dichotic pair was aligned on a single channel of audio tape using the Pulse Code Modulation system at the Haskin's Laboratories so that, when played stereophonically, competing syllables had a simultaneous onset. Thus, on every dichotic trial, each ear was simultaneously presented with a different syllable. This technique functionally lateralizes stimuli to the hemisphere contralateral to the stimulated ear when, as in the present case, there is sufficient stimulus competition (Springer et al., 1978; Sidtis, 1980).

Following verbal instructions, 10 binaural and $10 \mathrm{di}$ chotic practice trials were administered to familiarize the subject with both the stimuli and the procedure. Sixty experimental trials then were conducted. Subjects responded by writing the letters corresponding to the perceived sounds on an answer sheet and were encouraged to provide two answers on every trial. The preferred right hand was used in this standard test of interhemispheric auditory transfer. The results obtained in this way are virtually identical to those obtained with a spoken response.

The dichotic consonant-vowel discrimination test consisted of a modified version of the standard test previously described. In this version, a binaural test item was contained on audio tape $1 \mathrm{sec}$ after each dichotic pair. On half of the trials, the test item was the same as one of the preceding stimuli; on the remaining half, it was different. Left and right ear matches were equally likely. The test, which was administered twice at each session, consisted of four blocks of 28 trials. As with the standard test, verbal instructions were followed by binaural and dichotic practice trials after which the actual test was administered. The subjects responded manually, pointing to either a "yes" (indicating a match) or a "no" (indicating no match) response card. Hand of response was counterbalanced in an ABBA design. This test has been shown to elicit a significant left hemisphere advantage in normal subjects (J. J. Sidtis, submitted for publication).

Both dichotic tests were administered at approximately 78 dBA per channel using a Revox B-77 tape recorder and matched Telephonics TDH-39 earphones. The left-right orientation of the earphones was counterbalanced to control for any possible systematic channel differences.

The visual rhyming test was administered using the previously described microprocessor-video system. The form of the test was identical to that used in the semantic relations tests. Twenty-four high frequency nouns (AA or A) were presented twice, lateralized to one visual field or the other. Subjects chose the rhyming word (e.g., legegg; note-boat) from a response card with four alternatives using the hand homotopic to the stimulated field.

\section{Results}

Auditory testing. The results obtained from V. P. and J. W. on the standard test for interhemispheric auditory transfer are contained in Table II along with those obtained from a group of normal subjects. The left ear scores for both subjects were below those found in normal subjects, while their right ear scores were above the normal performance level. For J. W., preoperative testing was also conducted and accuracy on both left $(67 \%)$ and right $(77 \%)$ ear stimuli was within the normal range. Thus, these results demonstrate the classic effects of callosal section on the auditory system: The left ear stimuli are not transferred to the responding left hemisphere and, hence, are not reported by the subject. The

TABLE II

Accuracy of identification of consonant-vowel syllables lateralized to the left and right hemispheres following complete section of the corpus callosum

\begin{tabular}{lcc}
\hline \multirow{2}{*}{ Patient } & \multicolumn{2}{c}{ Hemisphere } \\
\cline { 2 - 3 } & Left & Right \\
\hline & \multicolumn{2}{c}{$\%$ correct } \\
V. P. & 100.0 & 8.0 \\
J. W. & 100.0 & 23.0 \\
Normal subjects $(N=10)$ & 78.0 & 65.0 \\
\hline
\end{tabular}


right ear stimuli, on the other hand, are processed by the left hemisphere without interference from the competing information normally transferred through the callosum (Sparks and Geschwind, 1968; Milner et al., 1968; Springer and Gazzaniga, 1975; Springer et al., 1978).

These results can be contrasted with those obtained when the left hand was used to provide a written response. When this was attempted out of vision so that the language-dominant, left hemisphere could not guide visually the left hand by ipsilateral control, V. P. performed at chance on both right (20\%) and left (17\%) ear stimuli, while J. W. refused to respond claiming that he could not move his left hand.

In order to evaluate phonetic discrimination in the right hemisphere independent of the capacity for linguistic expression, the dichotic speech sounds discrimination test was also administered. The results obtained from each subject are represented in Figure 1. With right hand responses (Fig. 1, $a$ and $c$ ), the pattern of asymmetry was identical to that found with the standard test requiring a written response: Near perfect performance on syllables presented to the right ear coupled with below chance performance on those presented to the left ear. For V.P., however, this pattern was reversed when the left hand was used to respond (Fig. 1b). Performance on left ear
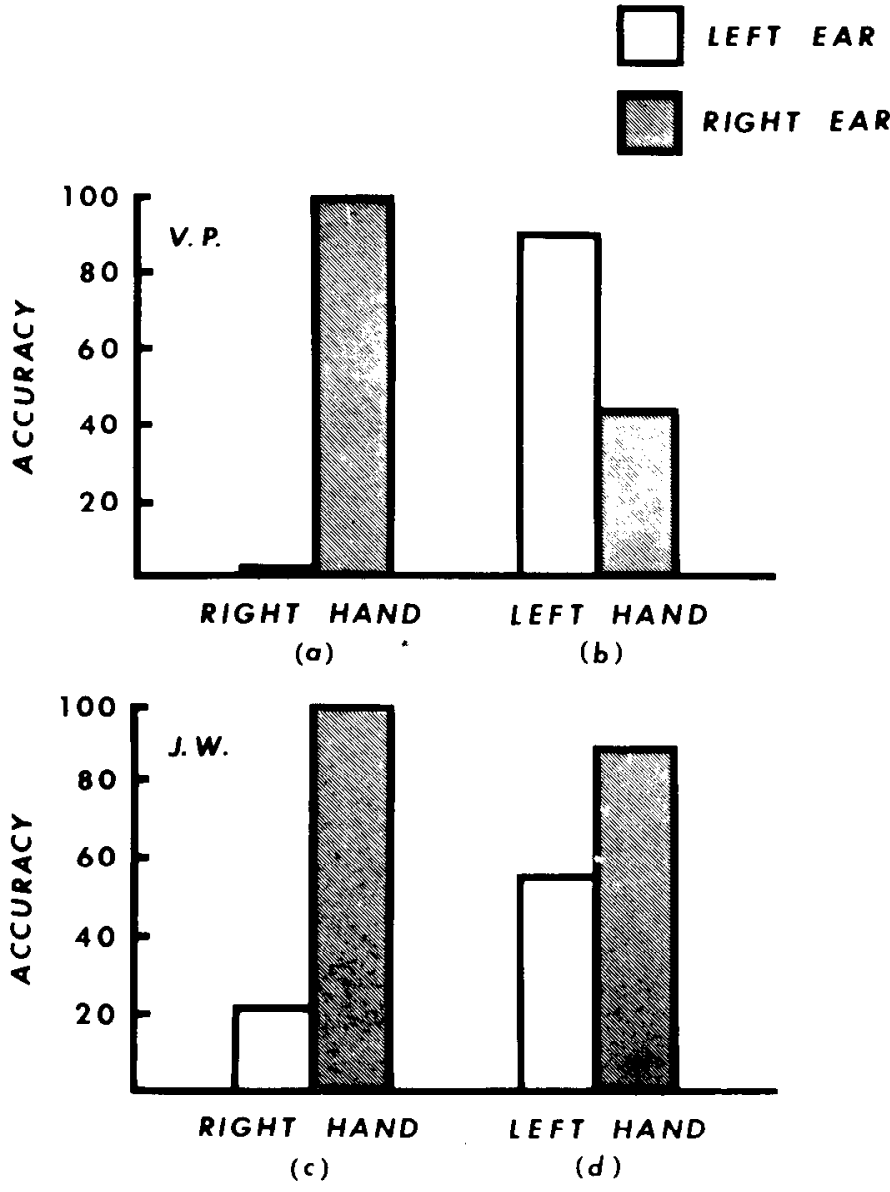

Figure 1. Accuracy of discrimination (percentage of correct responses) of consonant-vowel syllables as a function of ear of presentation and hand of response. Syllables presented to the right ear are lateralized to the left hemisphere, while those presented to the left ear are lateralized to the right hemisphere. speech sounds rose to a nearly perfect level, while right ear performance fell to chance. The interaction between hand of response and ear of presentation was highly significant $\left(\chi^{2}=29.70 ; p<0.001\right)$. This test was readministered approximately 6 weeks later and a similar reversal was observed $\left(\chi^{2}=79.12 ; p<0.001\right)$. No such reversal was observed for J. W. (Fig. 1d) on either the initial test or the follow-up administration some 12 weeks later and at no time was there a significant interaction between hand of response and ear of presentation. Although use of the left hand lowered his right ear performance, its effect was mainly seen on left ear accuracy where performance was significantly worse than chance with the right hand (binomial $p<0.05$ ) but not significantly different from chance with the left hand.

Because of the significant interaction on the speech discrimination test, V. P.'s ability to identify speech sounds lateralized to the right hemisphere was examined further by using the standard dichotic test with the response changed from writing to pointing. For this test, letters (B, D, G, P, T, and $\mathrm{K}$ ) corresponding to the auditory stimuli were presented on a choice card. In this test of identification, the interaction between response hand and ear of presentation was also apparent $\left(\chi^{2}=\right.$ $6.02 ; p<0.05$ ), although it was less pronounced than in the case of discrimination. The identification of right ear speech sounds was more accurate with a right hand response $(97 \%)$ than with a left hand response $(82 \%)$, while the opposite was true for left ear speech sounds ( $0 \%$ with the right hand, $17 \%$ with the left hand). Although the left ear-right hemisphere identification score improved with the use of the left hand, it did not increase to a level discernible from that expected by chance.

This form of the standard test was administered to $\mathrm{J}$. W. several months after the discrimination test was given. As in the discrimination test, there was no significant interaction between ear of stimulation and hand of response. Performance on right ear syllables was essentially identical to right (100\%) and left $(97 \%)$ hand responses. Left ear performance, on the other hand, had improved and was slightly better with the left hand (35\%) than with the right hand (27\%).

Visual testing. As with the auditory tests, V. P. and J. $W$. differed in their right hemisphere performance on the rhymes test. While the left hemispheres of both subjects performed at $100 \%$ accuracy, J. W.'s right hemisphere performance $(25 \%)$ was not significantly different from chance. Conversely, V. P.'s right hemisphere score was well above chance at $75 \%$ correct.

\section{Discussion}

The results of these auditory tests may be characterized in the following way. The standard test demonstrated both the absence of interhemispheric transfer and the ability of the left, but not the right, hemisphere to provide a written transcription following a dichotic trial. While these results appeared to show that only the left hemisphere in these subjects was capable of phonetic processing, this picture was altered in V. P. when the task was changed to discrimination rather than identification and a nonlinguistic response was allowed. Under these circumstances, V. P.'s right hemisphere perform- 
ance was nearly as accurate as that of her left hemisphere when each hemisphere's contralateral hand responses were compared. In contrast, J. W. showed no evidence of right hemisphere phonology under any of the test conditions.

The results of the visual tests also indicated that V.P. and $J . W$. differed in their right hemisphere abilities. Although either hemisphere in V. P. could use phonological rules to generate rhyme, only the left hemisphere in J. W. could perform this task. Thus, both auditory and visual tests indicated the presence of right hemisphere phonology in V. P. and suggested its absence in J. W.

The differences between V.P. and J. W. on these tests can be used to raise the question of whether hemispheric differences in language function are best characterized in qualitative or quantitative terms since the issue has been based largely on an apparent absence of phonology in right hemisphere language. While J. W.'s performance was typical of that used as evidence for a qualitative difference, V. P.'s performance provides a cautionary note: Care must be taken not to confuse performance and competence in the evaluation of the callosally disconnected hemisphere. As demonstrated in the case of manipulo-spatial tasks, the difference between an ability to perceive and an ability to respond can be critical in the evaluation of the capacity of an isolated hemisphere (LeDoux et al., 1977, 1978).

In terms of performance, the conditions under which V. P.'s right hemisphere was able to respond in these tests suggests a limited generative capacity compared to that found in the left hemisphere. Although her right hemisphere was proficient both in phonetic discrimination and in the use of phonetic rules for rhyming, in the competitive dichotic situation, it was clearly inferior to the left hemisphere in its capacity for generating linguistic as well as nonlinguistic responses. Pointing to one of two alternatives was accomplished by the right hemisphere, but neither pointing with six alternatives nor writing could be carried out by this hemisphere. It must be remembered, however, that performance on the auditory tests was achieved under conditions of double simultaneous stimulation so that every correct right hemisphere response represented an error to the left hemisphere. Thus, since both hemispheres received a different stimulus and both observed the response, the right hemisphere had to overcome the left hemisphere's dominance for language and probably its attempts to exert ipsilateral control over the responding left hand. These results, then, represent a conservative estimate of the right hemisphere's capacity to respond to linguistic information. In the next section, it will be shown that the limited generative capacity of V. P.'s right hemisphere language system can produce some linguistic expression through writing under conditions of unilateral rather than bilateral stimulation.

The performance of J.W. on these same tests provided no evidence of right hemisphere capacity for phonetic processing. There was no indication of competence so that performance is not an issue. It may well be that $\mathrm{J}$. W.'s right hemisphere is devoid of any phonological capacity. At the same time, it is important to consider the possibility that this hemisphere's linguistic capacity may have been insufficient to meet the demands of the present tests or to overcome the dominance of the left hemisphere system even in the context of unilateral stimulation. It is certainly possible that J. W.'s performance reflects the limited capacity of a weak phonological system. $^{3}$

The present results do not distinguish between the absence or presence, albeit in a weak form, of a right hemisphere phonological system in $J$. W. It must be emphasized, however, that although this patient's performance is comparable to that reported for other callosum-sectioned patients (Springer and Gazzaniga, 1975; Levy and Trevarthen, 1977; Zaidel, 1976a), it provides no basis for the postulation of novel right hemisphere language function. While such negative findings indicate a relative deficit, they are insufficient grounds for the claims of qualitatively different linguistic systems in each hemisphere.

\section{Some Observations on Generative Capacity}

In the previous section, it was suggested that the difference in right hemisphere language function between V. P. and J. W. could be characterized along the dimension of generative capacity. The present section offers further data with respect to this issue. First, there is a quantitative assessment of the ability of each hemisphere to execute verbal commands. Following this, observations of a more clinical nature are presented with respect to the right hemisphere's generation of writing and drawing.

\footnotetext{
${ }^{3}$ The perception of speech is viewed as a generative process in which the phonological coding of auditory information is governed by rules based on articulatory information (Liberman et al., 1967; Stevens, 1960; Stevens and House, 1972) and auditory memory (Oden and Massaro, 1978). It should be noted that, even in those approaches in which the generative rules underlying phonetic perception operate with reference to speech production, such rules can be used without accessing articulatory structures (Halle and Stevens, 1964; Liberman, 1957). The presence of rule-governed phonological processing in the right hemisphere, then, need not be excluded simply by virtue of this cortical system's usual inability to generate speech. If correspondence rules between speech articulation and perception are indeed necessary for phonetic processing, their presence in a right hemisphere language system would not be surprising given a history of callosal connection to the left hemisphere motor system and intact afferent connections with the articulatory system. One possibility, however, is that both articulatory information and auditory memory can provide input to generative phonological rules. Given the postoperative limitation on its data base, the right hemisphere language system could still generate phonological representations but would require greater capacity to do so. With an impoverished generative capacity, the successful generation of a phonetic representation may well require more context than is available in isolated syllables or, once generated, such representations may not be of significant functional saliency to warrant a response based on either component part or abstract rule as is necessary in the rhyming test. Such a situation could account for the apparent paradox in which the right hemisphere is able to comprehend words but unable to make phonological judgments. It should be noted that the inability to perform phonological judgments does not necessarily indicate the absence of a phonological system. Preschool children, for example, have difficulty with rhyme and alliteration, yet they are able to projuce and comprehend fluently (e.g., see Read, 1975).
} 


\section{Methods}

\section{Materials and procedures}

Forty-five commands (e.g., smile, clap, rub, kick) were presented twice, lateralized to one visual field or the other using the microprocessor-video system. Subjects were instructed to carry out the action conveyed by the word flashed on the video screen. Observations of the right hemisphere's writing and drawing ability were made during various lateralized picture and word tests conducted over several sessions.

\section{Results and Discussion}

Commands. Patient V. P., like patient P. S., demonstrated a clear bilateral ability to follow verbal commands (right hemisphere accuracy, $80.0 \%$; left hemisphere accuracy, $85.0 \%$ ). As noted earlier, except for P. S., such right hemisphere performance has not been observed in other patients found to have right hemisphere language. J. W.'s right hemisphere performance $(42.5 \%)$ falls in an intermediate range being better than chance but inferior to that found in the left $(95.3 \%)$. Taken as a measure of generative ability, these data provide further evidence that such capacity does not necessarily accompany right hemisphere receptive function, but may vary in the degree to which it is present. The issue of praxis and callosal section is discussed in more detail elsewhere (B. T. Volpe, J. J. Sidtis, J. D. Holtzman, D. H. Wilson and M. S. Gazzaniga, manuscript in preparation).

Writing. Over the course of several test sessions, it was observed that, following left field stimulation, V.P. could report the stimulus by writing with her left hand. When such responses were made out of vision, their content remained unknown to the left hemisphere language system; upon questioning, she could not provide information about what she had written. Her right hemisphere was capable of writing following not only the presentation of word stimuli but also following the presentation of pictures. Thus, her writing reflected more than just the ability to retain and copy words. With further testing, it was also found that her left hand could express the answers to questions posed about right hemisphere stimuli.

In contrast with the results obtained during the competitive dichotic testing, V. P. demonstrated that her right hemisphere was indeed capable of producing linguistic responses when the stimulation was unilateral and the left hemisphere was unable to observe the response. Under these conditions, there was presumably less interference from the dominant hemisphere and hence, the right hemisphere's capacity was sufficient for linguistic expression.

J. W., on the other hand, demonstrated no evidence of right hemisphere writing. At best, he would produce an unrelated letter or two with his left hand, never a complete word, and usually nothing more than a few disconnected lines. His ability to name the letters produced out of vision on such trials strongly suggested that the source of such writing was the left hemisphere rather than the right, mediated by ipsilateral control.

Drawing. Although unable to write following left field stimulation, J. W.'s performance on the verbal commands suggested that his right hemisphere was not completely agenerative. This suggestion was supported by his ability to use his left hand to draw following the presentation of pictorial stimuli to the left visual field. As was the case with V. P.'s writing, J. W.'s right hemisphere drawing, when executed out of vision, did not provide information to the left hemisphere. In fact, when confronted with readily identifiable sketches produced by the right hemisphere, the left hemisphere expressive language system named the referent with great reluctance, often claiming that such sketches were "just scribble." Similar sketches produced by the left hemisphere were readily named.

These observations further demonstrate the differences in the generative capacities of these right hemisphere language systems. V. P.'s right hemisphere had a greater linguistic generative capacity than that of J. W., allowing it to express itself more effectively in carrying out verbal commands and in writing. The generative limitation of J. W.'s right hemisphere did not appear to be global, however, since it was capable of executing drawings. Rather, it seemed to reflect limits in its language function.

\section{General Discussion}

Several points can be made about the nature of right hemisphere language. Based on evidence obtained from the commissurotomy population, it must be stated that right hemisphere linguistic representation is uncommon. Moreover, the extent of right hemisphere language is variable across patients who possess it. At one extreme, there is the apparent ability to generate speech while at the other, there appears to be only word comprehension with little expressive ability. The present results have suggested that the differences between left and right hemisphere language systems are quantitative rather than qualitative and that such qualitative differences can be characterized along a continuum of generative capacity. Variability in the degree of this generative capacity is believed to be responsible for differences observed across right hemisphere language systems.

The variability in the extent of right hemisphere language observed in V. P. and J. W. reflects the range of function found across the commissurotomy population and is critical to understanding the nature of this function. If a special right hemisphere language system is postulated for patients at one end of the performance range, yet another must be posed for patients whose performance lies at the other end. Rather than suggesting that the right hemisphere is a spawning ground for novel mental systems, it has been argued that performance variability reflects differences in capacity that are more obvious in some tasks (e.g., phonetic processing) than in others (e.g., semantic processing): Why this may be has yet to be determined.

Within the population of commissurotomy patients with right hemisphere language, J. W. appears to be similar to patients in the earlier Bogen and Vogel commissurotomy series (L. B. and N. G.; Gazzaniga and Sperry, 1967) who also demonstrated right hemisphere 
language comprehension in the absence of the ability to respond to phonological relationships. In V. P., on the other hand, there was evidence of both phonological processing and a greater linguistic generative capacity in the right hemisphere than was seen in J. W. Thus, she was more like P. S. than like J. W., L. B., or N. G. V. P.'s right hemisphere execution of verbal commands and her generation of written responses were comparable to those abilities observed in P. S. during the first two postoperative years. The similarly high level of generative capacity in V.P. and P. S. suggests that the language in V.P.'s right hemisphere, as in that of P. S., represents a dynamic cognitive system. On the basis of her right hemisphere's expressive capacity and its ability to use phonetic information, we predict that she too will develop the capacity for right hemisphere speech. ${ }^{4}$

Finally, it must be added that the reasons for variability in right hemisphere language are unclear, but several factors deserve note. First, it may be that such variability represents normal functional variation within the righthanded population. There is no independent way to evaluate the probability of this, however, since clinical series of patients who have undergone the Wada test and of patients with aphasia deal, respectively, with dominant language lateralization and recovery of function. A second reason finds some support in the clinical histories of most of the patients. That is, the presence of early left hemisphere pathology is likely to result in a bilateralization of language function (Hecaen and Albert, 1978). There is some evidence that such a condition was present in at least four of the relevant patients (V. P., P. S., N. G., and L. B.).

Little can be said about two other factors, although they deserve some mention. Two of the patients with right hemisphere language are women (V. P. and N. G.), and it has been suggested that females are more likely to have a bilateral language organization than are men (McGlone, 1977). Two of the patients (V. P. and J. W.) underwent callosal section in stages. This procedure may influence functional reorganization in the interoperative period in some way analogous to the presumed reorganization that underlies the behavioral differences observed between serial and nonserial cortical lesions (Reitz, 1969). The sample size is currently too small to evaluate the significance of either gender or serial versus complete callosal section. At present, it appears that early pathology is the most likely factor in influencing the presence and extent of right hemisphere language.

The evaluation of the nature of right hemisphere language is at an early stage, but it appears that such function represents an incomplete and weaker form of

\footnotetext{
${ }^{4}$ Our most recent evaluation of V. P.'s right hemisphere performance suggests that she is indeed developing the capacity for right hemisphere speech. At our first evaluation (4 months postoperative), she could not name any stimuli presented to her left sensory field. By the 12th postoperative month, however, she named $32 \%$ of the left visual field words presented in one test. After such naming occurred, she often expressed surprise at these responses and demonstrated no insight as to why they were emitted. At this time, V. P.'s performance on a samedifferent interfield comparison test for visual transfer was at chance, further suggesting that such verbalizations were the product of right hemisphere cognition.
}

the left hemisphere system. Future research will attempt to clarify the apparent generative differences between these systems and to investigate the degree to which generative capacity in one cognitive system is independent of that found in other cognitive systems within a callosally disconnected hemisphere.

\section{References}

Gazzaniga, M. S. (1970) The Bisected Brain, Appleton-Century-Crofts, New York.

Gazzaniga, M. S., and S. A. Hillyard (1971) Language and speech capacity of the right hemisphere. Neuropsychologia 9: $273-280$

Gazzaniga, M. S., and J. E. LeDoux (1978) The Integrated Mind, Plenum Press, New York.

Gazzaniga, M. S., and R. W. Sperry (1967) Language after section of the cerebral commissures. Brain 90: 131-148.

Gazzaniga, M. S., J. E. Bogen, and R. W. Sperry (1967) Dyspraxia following division of the cerebral hemispheres. Arch. Neurol. 16: 606-612.

Gazzaniga, M. S., J. E. LeDoux, and D. H. Wilson (1977) Language, praxis, and the right hemisphere: Clues to some mechanisms of consciousness. Neurology (N. Y.) 27: 11441147.

Gazzaniga, M. S., B. T. Volpe, C. S. Smylie, D. H. Wilson, and J. E. LeDoux (1979) Plasticity in speech organization following commissurotomy. Brain 102: 805-815.

Halle, M., and K. N. Stevens (1964) Speech recognition: A model and a program for research. In The Structure of Language, J. A. Fodor and J. J. Katz, eds., pp. 604-612, Prentice Hall, Englewood Cliffs, NJ.

Hecaen, H. (1978) Right hemisphere contribution to language function. In Cerebral Correlates of Conscious Experience, P. A. Buser and A. Rougeal-Buser, eds., pp. 199-214, NorthHolland, New York.

Hecaen, H., and M. L. Albert (1978) Human Neuropsychology, John Wiley and Sons, New York.

LeDoux, J. E., D. H. Wilson, and M. S. Gazzaniga (1977) Manipulo-spatial aspects of cerebral lateralization. Neuropsychologia 15: 743-750.

LeDoux, J. E., D. H. Wilson, and M. S. Gazzaniga (1978) Block design performance following callosal sectioning. Arch. Neurol. 35: 506-508.

Levy, J., and C. Trevarthen (1977) Perceptual, semantic and phonetic aspects of elementary language processes in splitbrain patients. Brain 100: 105-118.

Liberman, A. M. (1957) Results of research on speech perception. J. Acoust. Soc. Am. 29: 117-123.

Liberman, A. M., F. S. Cooper, D. S. Shankweiler, and M. Studdert-Kennedy (1967) Perception of the speech code. Psychol. Rev. 74: 431-461.

McGlone, J. (1977) Sex differences in the cerebral organization of verbal functions in patients with unilateral brain lesions. Brain 100: 775-793.

Milner, B., L. Taylor, and R. W. Sperry (1968) Lateralized suppression of dichotically presented digits after commissural section in man. Science 161: 184-186.

Oden, G. C., and D. W. Massaro (1978) Integration of featural information in speech. Psychol. Rev. 85: 172-191.

Kead, C. (1975) The preschool orthographer. In Foundations of Language Development, E. H. Lenneberg and E. Lenneberg, eds., Vol. 2, pp. 329-346, Academic Press, New York.

Reitz, S. L. (1969) Effects of serial disconnection of striate and temporal cortex on visual discrimination performance in monkeys. J. Comp. Physiol. Psychol. 68: 139-146.

Sidtis, J. J. (1980) The complex tone test: Implications for the 
assessment of auditory laterality effects. Neuropsychologia, in press.

Sparks, R., and N. Geschwind (1968) Dichotic listening in man after section of the neocortical commissures. Cortex 4: 3-16.

Springer, S. P., and M. S. Gazzaniga (1975) Dichotic testing of partial and complete split-brain subjects. Neuropsychologia 13: 341-346.

Springer, S. P., J. J. Sidtis, D. H. Wilson, and M. S. Gazzaniga (1978) Left ear performance in dichotic listening following commissurotomy. Neuropsychologia 16: 305-312.

Stevens, K. N. (1960) Toward a model for speech recognition. J. Acoust. Soc. Am. 32: 47-55.

Stevens, K. N., and A. S. House (1972) Speech perception. In Foundations of Modern Auditory Theory, J. Tobias, ed., Vol. II, pp. 1-62, Academic Press, New York.

Thorndike, E. L., and I. Lorge (1944) The Teacher's Word
Book of 30,000 Words, Teachers College Bureau of Publications, Columbia University, New York.

Wilson, D. H., A. Reeves, and M. S. Gazzaniga (1978) Division of the corpus callosum for uncontrollable epilepsy. Neurology (N. Y.) 28: 649-653.

Zaidel, E. (1976a) Auditory vocabulary of the right hemisphere following brain bisection or hemidecortication. Cortex 12: 191-211.

Zaidel, E. (1976b) Language, dichotic listening and the disconnected hemispheres. In Conference on Human Brain Function, D. O. Walter, L. Rogers, and J. M. Finzi-Fried, eds., pp. 87-93, Brain Information Service/BRI Publications Office, UCLA, Los Angeles.

Zaidel, E. (1977) Unilateral auditory language comprehension on the token test following cerebral commissurotomy and hemispherectomy. Neuropsychologia 15: 1-18. 